\title{
Hydrodynamic limit for perturbation of a hyperbolic equilibrium point in two-component systems
}

\author{
Benedek Valkó ${ }^{\mathrm{a}, \mathrm{b}}$ \\ a Alfréd Rényi Institute of Mathematics, Hungarian Academy of Sciences, Reáltanoda u. 13-15, H-1053, Budapest, Hungary \\ ${ }^{\mathrm{b}}$ Technical University Budapest, Institute of Mathematics, Egry József u. 1, H-1111 Budapest, Hungary
}

Received 3 February 2004; received in revised form 26 October 2004; accepted 28 January 2005

Available online 26 September 2005

\begin{abstract}
We consider one-dimensional, locally finite interacting particle systems with two conservation laws. The models have a family of stationary measures with product structure and we assume the existence of a uniform bound on the inverse of the spectral gap which is quadratic in the size of the system. Under Eulerian scaling the hydrodynamic limit for the macroscopic density profiles leads to a two-component system of conservation laws. The resulting pde is hyperbolic inside the physical domain of the macroscopic densities, with possible loss of hyperbolicity at the boundary.

We investigate the propagation of small perturbations around a hyperbolic equilibrium point. We prove that the perturbations essentially evolve according to two decoupled Burgers equations. The scaling is not Eulerian: if the lattice constant is $n^{-1}$, the perturbations are of order $n^{-\beta}$ then time is speeded up by $n^{1+\beta}$. Our derivation holds for $0<\beta<\frac{1}{5}$. The proof relies on Yau's relative entropy method, thus it applies only in the regime of smooth solutions.

This result is an extension of [T. Seppäläinen, Perturbation of the equilibrium for a totally asymmetric stick process in one dimension, Ann. Probab. 29 (2001) 176-204] and [B. Tóth, B. Valkó, Between equilibrium fluctuations and Eulerian scaling. Perturbation of equilibrium for a class of deposition models, J. Statist. Phys. 109 (2002) 177-205] where the analogue result was proved for systems with one conservation law. It also complements [B. Tóth, B. Valkó, Perturbation of singular equilibria of hyperbolic two-component systems: a universal hydrodynamic limit, Commun. Math. Phys. 256 (2005) 111-157] where it was shown that perturbations around a nonhyperbolic boundary equilibrium point are driven by a universal two-by-two system of conservation laws.

(c) 2005 Elsevier SAS. All rights reserved.

\section{Résumé}

Nous considérons un système localement fini de particules interagissantes à deux lois de conservation en une dimension. Les modèles possédent une famille de mesures stationnaires de structure produit et nous supposons qu'il existe une borne uniforme pour l'inverse du trou spectral qui est quadratique en la taille du système. La limite hydrodynamique pour le profil de densité microscopique mène à un système de lois de conservation à deux composantes. L'EDP obtenue est hyperbolique à l'intérieur du domaine physique des densités macroscopiques avec perte éventuelle de l'hyperbolicité à la frontière.
\end{abstract}

E-mail address: valko@renyi.hu (B. Valkó). 
Nous étudions la propagation de petites perturbations autour d'un point d'équilibre hyperbolique. Nous démontrons que les perturbations évoluent essentiellement selon deux équations de Burgers découplées. L'échelle n'est pas eulerienne : si la constante du treillis est $n^{-1}$ et les perturbations sont d'ordre $n^{-\beta}$, alors le temps est accéléré par un facteur $n^{1+\beta}$. Notre dérivation est valable pour $0<\beta<\frac{1}{5}$. La preuve s'appuie et non pas sáffmie sur la méthode d'entropie relative de Yau, et s'applique donc seulement au régime des solutions lisses.

Ce résultat est une extension de [T. Seppäläinen, Perturbation of the equilibrium for a totally asymmetric stick process in one dimension, Ann. Probab. 29 (2001) 176-204] et [B. Tóth, B. Valkó, Between equilibrium fluctuations and Eulerian scaling. Perturbation of equilibrium for a class of deposition models, J. Statist. Phys. 109 (2002) 177-205] où un résultat analogue a été démontré pour des systèmes à une loi de conservation. Il complète également [B. Tóth, B. Valkó, Perturbation of singular equilibria of hyperbolic two-component systems: a universal hydrodynamic limit, Commun. Math. Phys. 256 (2005) 111-157] où il est montré que les perturbations autour d'un point d'équilibre frontière non-hyperbolique sont conduites par un système universel de lois de conservations.

(C) 2005 Elsevier SAS. All rights reserved.

MSC: 60K35; 35L65

Keywords: Hydrodynamic limit; Relative entropy; Hyperbolic systems of conservation laws

\section{Introduction}

There are several results dealing with the perturbation analysis of hydrodynamic limits for interacting particle systems. In the landmark paper [5] the authors prove that for the asymmetric simple exclusion, in dimensions higher than 2, perturbations of order $n^{-1}$ of a constant profile evolve according to a certain parabolic equation under diffusive scaling (time rescaled by $n^{2}$, space by $n^{-1}$ ). It is well-known, that under Eulerian scaling (time rescaled by $n$, space by $n^{-1}$ ) the hydrodynamic limit leads to a hyperbolic conservation law (the Burgers equation), the perturbation limit gives the same equation with the Navier-Stokes correction. (For a survey on the microscopic interpretations of the Navier-Stokes equations see the end of Chapter 7 of [8].)

Motivated by [5] T. Seppäläinen investigated a similar problem in one dimension for the so-called totally asymmetric stick process. In [13] he proves that a perturbation of order $n^{-\beta}$ of the constant profile is governed by the Burgers equation (even after the appearance of shocks) if time is rescaled by $n^{1+\beta}$ and space by $n^{-1}$, where $\beta \in\left(0, \frac{1}{2}\right)$ is a fixed constant. Independently, in [14] the authors partially extend this result by proving that one gets universally the Burgers equation in the hydrodynamic limit for similar perturbations of equilibrium for a wide class of one-dimensional interacting particle systems with one conservation law. The models are not reversible and not necessarily attractive. The proof relies on H.T. Yau's relative entropy method, it only applies in the smooth regime of solutions and it only works for $\beta \in\left(0, \frac{1}{5}\right)$. It is conjectured that the result should hold for all $\beta \in\left(0, \frac{1}{2}\right)$ even without the smoothness condition as in the result of [13].

This universal result may be explained by the following arguments. Under Eulerian scaling these systems admit in the hydrodynamic limit a hyperbolic conservation law of the form

$$
\partial_{t} u+\partial_{x} J(u)=0 .
$$

Taking a point $u_{0}$ with $J^{\prime \prime}\left(u_{0}\right) \neq 0$ simple (although formal) calculations yield that solutions of (1), with initial conditions which are small perturbations $u_{0}$, are governed by the Burgers equation. See [14] for the 'more precise' formulation.

In the present paper we give an extension of the results of $[13,14]$ for systems with 2 conserved quantities. In [15] a general one-dimensional family of lattice-models was introduced. The models are locally finite interacting particle systems with two conservation laws which possess a family of stationary measures with product structure. 
In that paper it is shown (in the regime of smooth solutions) that in Eulerian scaling we get a hydrodynamic limit of the form

$$
\left\{\begin{array}{l}
\partial_{t} u+\partial_{x} \Phi(u, v)=0 \\
\partial_{t} v+\partial_{x} \Psi(u, v)=0
\end{array}\right.
$$

where $(u, v) \in \mathcal{D}$ and $\mathcal{D}$ is a convex compact polygon (the physical domain, see (6) for the definition). We note, that [10] gives the first major result about the Eulerian hydrodynamic limit for multi-component hyperbolic systems, namely for Hamiltonian systems perturbed by a weak noise. In [15] it was also shown that an Onsager-type symmetry relation holds for the macroscopic flux functions $\Phi, \Psi$ (see Lemma 1). One of the consequences of this relation is that inside the physical domain $\mathcal{D}$ the pde (2) is (weakly) hyperbolic, i.e. the Jacobian can be diagonalized in the real sense. Experience shows, that the limiting pde is strongly hyperbolic (the Jacobian has two distinct real eigenvalues) in the whole physical domain except some special points on the boundary $\partial \mathcal{D}$.

We consider perturbations of order $n^{-\beta}$ around a constant equilibrium point $\left(u_{0}, v_{0}\right) \in \mathcal{D}$, which is strictly hyperbolic. We prove that rescaling time by $n^{1+\beta}$ and space by $n^{-1}$ the evolution of the perturbations are governed by two decoupled equations. These are 'usually' Burgers equations, see the remark at the end of Subsection 3.1. This result agrees with the formal perturbation of the pde (2) e.g. with the method of weakly nonlinear geometric optics (see [4,7]).

The reason for the decoupling of the resulting pde system is the strict hyperbolicity, basically, the two different eigenvalues (sound speeds) cause the equations to separate. In the paper [16] perturbation around a special nonhyperbolic point was considered in a similar setting, it was proved that in that case in the limit the evolution obeys a two-by-two system of conservation laws which cannot be decoupled. The treatment of that problem needs more complex tools than our proofs, sophisticated pde methods are used besides Yau's method.

Our proof follows the relative entropy method using similar steps as [14] (thus it only applies in the regime of smooth solutions), but it also heavily relies on the Onsager-type symmetry relation proved in [15]. We assume the existence of a uniform bound on the inverse of the spectral gap, quadratic in system size, to be able to prove the so-called one block estimate. We do not deal with the proof of the spectral gap bound, but we remark that with the techniques of [9] one should be able to get the desired gap estimates for a large class of systems. Our result holds for $\beta \in\left(0, \frac{1}{5}\right)$. Assuming the stronger (but harder to prove) logarithmic-Sobolev bound we get the result for $\beta \in\left(0, \frac{1}{3}\right)$.

\section{Microscopic models}

We consider the family of microscopic models investigated in [15]. We go over the definitions and the important properties, for the details we refer the reader to the original paper. We also give a brief description of two specific models (introduced in the same paper).

\subsection{State space, conserved quantities, generator}

Throughout this paper we denote by $\mathbb{T}^{n}$ the discrete tori $\mathbb{Z} / n \mathbb{Z}, n \in \mathbb{N}$, and by $\mathbb{T}$ the continuous torus $\mathbb{R} / \mathbb{Z}$. We will denote the local spin state by $\Omega$, we only consider the case when $\Omega$ is finite. The state space of the interacting particle system is

$$
\Omega^{n}:=\Omega^{\mathbb{T}^{n}} .
$$

Configurations will be denoted

$$
\underline{\omega}:=\left(\omega_{j}\right)_{j \in \mathbb{T}^{n} \in \Omega^{n} .}
$$

The two conserved quantities are denoted by

$$
\zeta: \Omega \rightarrow \mathbb{Z}, \quad \eta: \Omega \rightarrow \mathbb{Z},
$$


we also use the notations $\zeta_{j}=\zeta\left(\omega_{j}\right), \eta_{j}=\eta\left(\omega_{j}\right)$. We assume that the conserved quantities are different and nontrivial, i.e. the functions $\zeta, \eta$ and the constant function 1 on $\Omega$ are linearly independent.

We consider the rate function $r: \Omega \times \Omega \times \Omega \times \Omega \rightarrow \mathbb{R}_{+}$. The dynamics of the system consists of elementary jumps effecting nearest neighbor spins, $\left(\omega_{j}, \omega_{j+1}\right) \rightarrow\left(\omega_{j}^{\prime}, \omega_{j+1}^{\prime}\right)$, performed with rate $r\left(\omega_{j}, \omega_{j+1} ; \omega_{j}^{\prime}, \omega_{j+1}^{\prime}\right)$.

We require that the rate function $r$ satisfy the following conditions:

(A) If $r\left(\omega_{1}, \omega_{2} ; \omega_{1}^{\prime}, \omega_{2}^{\prime}\right)>0$ then

$$
\begin{aligned}
& \zeta\left(\omega_{1}\right)+\zeta\left(\omega_{2}\right)=\zeta\left(\omega_{1}^{\prime}\right)+\zeta\left(\omega_{2}^{\prime}\right), \\
& \eta\left(\omega_{1}\right)+\eta\left(\omega_{2}\right)=\eta\left(\omega_{1}^{\prime}\right)+\eta\left(\omega_{2}^{\prime}\right) .
\end{aligned}
$$

This means that $\zeta$ and $\eta$ are indeed conserved quantities.

(B) For every $Z \in[n \min \zeta, n \max \zeta] \cap \mathbb{Z}, N \in[n \min \eta, n \max \eta] \cap \mathbb{Z}$ the set

$$
\Omega_{Z, N}^{n}:=\left\{\underline{\omega} \in \Omega^{n}: \sum_{j \in \mathbb{T}^{n}} \zeta_{j}=Z, \sum_{j \in \mathbb{T}^{n}} \eta_{j}=N\right\}
$$

is an irreducible component of $\Omega^{n}$, i.e. if $\underline{\omega}, \underline{\omega}^{\prime} \in \Omega_{Z, N}^{n}$ then there exists a series of elementary jumps with positive rates transforming $\underline{\omega}$ into $\underline{\omega}^{\prime}$. This ensures that there are no hidden conservation laws.

(C) There exists a probability measure $\pi$ on $\Omega$ which puts positive mass on each element of $\Omega$ and for any $\omega_{1}$, $\omega_{2}, \omega_{3} \in \Omega$

$$
Q\left(\omega_{1}, \omega_{2}\right)+Q\left(\omega_{2}, \omega_{3}\right)+Q\left(\omega_{3}, \omega_{1}\right)=0,
$$

where

$$
Q\left(\omega_{1}, \omega_{2}\right):=\sum_{\omega_{1}^{\prime}, \omega_{2}^{\prime} \in \Omega}\left\{\frac{\pi\left(\omega_{1}^{\prime}\right) \pi\left(\omega_{2}^{\prime}\right)}{\pi\left(\omega_{1}\right) \pi\left(\omega_{2}\right)} r\left(\omega_{1}^{\prime}, \omega_{2}^{\prime} ; \omega_{1}, \omega_{2}\right)-r\left(\omega_{1}, \omega_{2} ; \omega_{1}^{\prime}, \omega_{2}^{\prime}\right)\right\} .
$$

This condition will imply that the measure $\prod_{j \in \mathbb{T}^{n}} \pi$ is stationary for our process on $\Omega^{n}$.

For a precise formulation of the infinitesimal generator on $\Omega^{n}$ we first define the map $\Theta_{j}^{\omega^{\prime}, \omega^{\prime \prime}}: \Omega^{n} \rightarrow \Omega^{n}$ for every $\omega^{\prime}, \omega^{\prime \prime} \in \Omega, j \in \mathbb{T}^{n}$ :

$$
\left(\Theta_{j}^{\omega^{\prime}, \omega^{\prime \prime}} \underline{\omega}\right)_{i}= \begin{cases}\omega^{\prime} & \text { if } i=j \\ \omega^{\prime \prime} & \text { if } i=j+1 \\ \omega_{i} & \text { if } i \neq j, j+1\end{cases}
$$

The infinitesimal generator of the process defined on $\Omega^{n}$ is

$$
L^{n} f(\underline{\omega})=\sum_{j \in \mathbb{T}^{n}} \sum_{\omega^{\prime}, \omega^{\prime \prime} \in \Omega} r\left(\omega_{j}, \omega_{j+1} ; \omega^{\prime}, \omega^{\prime \prime}\right)\left(f\left(\Theta_{j}^{\omega^{\prime}, \omega^{\prime \prime}} \underline{\omega}\right)-f(\underline{\omega})\right) .
$$

We denote by $\mathcal{X}_{t}^{n}$ the Markov process on the state space $\Omega^{n}$ with infinitesimal generator $L^{n}$.

\subsection{Stationary measures}

For every $\theta, \tau \in \mathbb{R}$ let $G(\theta, \tau)$ be the moment generating function defined below:

$$
G(\theta, \tau):=\log \sum_{\omega \in \Omega} \mathrm{e}^{\theta \zeta(\omega)+\tau \eta(\omega)} \pi(\omega) .
$$

We define the probability measures

$$
\pi_{\theta, \tau}(\omega):=\pi(\omega) \exp (\theta \zeta(\omega)+\tau \eta(\omega)-G(\theta, \tau))
$$


on $\Omega$. Using condition (C), by very similar considerations as in the papers $[1,3,12,14]$ one can show that for any $\theta, \tau \in \mathbb{R}$ the product measure

$$
\pi_{\theta, \tau}^{n}:=\prod_{j \in \mathbb{T}^{n}} \pi_{\theta, \tau}
$$

is stationary for the Markov process $\mathcal{X}_{t}^{n}$ on $\Omega^{n}$ with infinitesimal generator $L^{n}$. We will refer to these measures as the canonical measures. Since $\sum_{j} \zeta_{j}$ and $\sum_{j} \eta_{j}$ are conserved, the canonical measures on $\Omega^{n}$ are not ergodic. The conditioned measures defined on $\Omega_{Z, N}^{n}$ by:

$$
\pi_{Z, N}^{n}(\underline{\omega}):=\pi_{\theta, \tau}^{n}\left(\underline{\omega} \mid \sum_{j} \zeta_{j}=Z, \sum_{j} \eta_{j}=N\right)=\frac{\pi_{\theta, \tau}^{n}(\underline{\omega}) \mathbb{1}\left\{\underline{\omega} \in \Omega_{Z, N}^{n}\right\}}{\pi_{\theta, \tau}^{n}\left(\Omega_{Z, N}^{n}\right)}
$$

are also stationary and due to condition (B) satisfied by the rate functions they are also ergodic. We shall call these measures the microcanonical measures of our system. It is easy to see that the measure $\pi_{Z, N}^{n}$ does not depend on the values of $\theta, \tau$.

\subsection{Expectations, fluxes}

Expectation, variance, covariance with respect to the measures $\pi_{\theta, \tau}^{n}$ will be denoted by $\mathbf{E}_{\theta, \tau}(\cdot), \operatorname{Var}_{\theta, \tau}(\cdot)$, $\operatorname{Cov}_{\theta, \tau}(\cdot)$.

We compute the expectations of the conserved quantities with respect to the canonical measures, as functions of the parameters $\theta$ and $\tau$ :

$$
\begin{aligned}
& u(\theta, \tau):=\mathbf{E}_{\theta, \tau}(\zeta)=\sum_{\omega \in \Omega} \zeta(\omega) \pi_{\theta, \tau}(\omega)=\partial_{\theta} G(\theta, \tau)=G_{\theta}, \\
& v(\theta, \tau):=\mathbf{E}_{\theta, \tau}(\eta)=\sum_{\omega \in \Omega} \eta(\omega) \pi_{\theta, \tau}(\omega)=\partial_{\tau} G(\theta, \tau)=G_{\tau} .
\end{aligned}
$$

We will usually note partial derivatives by using the respective subscripts, as long as it does not cause confusion. Elementary calculations show, that the matrix-valued function

$$
\left(\begin{array}{cc}
u_{\theta} & u_{\tau} \\
v_{\theta} & v_{\tau}
\end{array}\right)=\left(\begin{array}{ll}
G_{\theta \theta} & G_{\theta \tau} \\
G_{\theta \tau} & G_{\tau \tau}
\end{array}\right)=: G^{\prime \prime}(\theta, \tau)
$$

is equal to the covariance matrix $\operatorname{Cov}_{\theta, \tau}(\zeta, \eta)$ and as a consequence the function $(\theta, \tau) \mapsto(u(\theta, \tau), v(\theta, \tau))$ is invertible. We denote the inverse function by $(u, v) \mapsto(\theta(u, v), \tau(u, v))$. Denote by $(u, v) \mapsto S(u, v)$ the convex conjugate (Legendre transform) of the strictly convex function $(\theta, \tau) \mapsto G(\theta, \tau)$ :

$$
S(u, v):=\sup _{\theta, \tau}(u \theta+v \tau-G(\theta, \tau)),
$$

and

$$
\mathcal{D}:=\overline{\{(u, v) \in \mathbb{R} \times \mathbb{R}: S(u, v)<\infty\}}=\operatorname{co}\{(\zeta(\omega), \eta(\omega)): \omega \in \Omega\},
$$

where co stands for convex hull and $\bar{A}$ is the closure of $A$. In probabilistic terms: $S(u, v)$ is the rate function for joint large deviations of $\left(\sum_{j} \zeta_{j}, \sum_{j} \eta_{j}\right)$. If $(u, v)$ is inside $\mathcal{D}$ then we have

$$
\theta(u, v)=S_{u}(u, v), \quad \tau(u, v)=S_{v}(u, v) .
$$

With slight abuse of notation we shall denote:

$$
\pi_{\theta(u, v), \tau(u, v)}=: \pi_{u, v}, \quad \pi_{\theta(u, v), \tau(u, v)}^{n}=: \pi_{u, v}^{n}, \quad \mathbf{E}_{\theta(u, v), \tau(u, v)}=: \mathbf{E}_{u, v}, \quad \text { etc. }
$$


Clearly, $\pi_{u, v}$ extends naturally onto the boundary of $\mathcal{D}$, in that case $\pi_{u, v}$ puts zero weight on some of the elements of $\Omega$.

We introduce the flux of the conserved quantities. The infinitesimal generator $L^{n}$ acts on the conserved quantities as follows:

$$
\begin{aligned}
& L^{n} \zeta_{i}=-\phi\left(\omega_{i}, \omega_{i+1}\right)+\phi\left(\omega_{i-1}, \omega_{i}\right)=:-\phi_{i}+\phi_{i-1}, \\
& L^{n} \eta_{i}=-\psi\left(\omega_{i}, \omega_{i+1}\right)+\psi\left(\omega_{i-1}, \omega_{i}\right)=:-\psi_{i}+\psi_{i-1},
\end{aligned}
$$

where

$$
\begin{aligned}
\phi\left(\omega_{1}, \omega_{2}\right) & :=\sum_{\omega_{1}^{\prime}, \omega_{2}^{\prime} \in \Omega} r\left(\omega_{1}, \omega_{2} ; \omega_{1}^{\prime}, \omega_{2}^{\prime}\right)\left(\zeta\left(\omega_{2}^{\prime}\right)-\zeta\left(\omega_{2}\right)\right)+C_{1}, \\
\psi\left(\omega_{1}, \omega_{2}\right) & :=\sum_{\omega_{1}^{\prime}, \omega_{2}^{\prime} \in \Omega} r\left(\omega_{1}, \omega_{2} ; \omega_{1}^{\prime}, \omega_{2}^{\prime}\right)\left(\eta\left(\omega_{2}^{\prime}\right)-\eta\left(\omega_{2}\right)\right)+C_{2} .
\end{aligned}
$$

The constants $C_{1}, C_{2}$ may be chosen arbitrarily, we will fix them later. We shall denote the expectations of these functions with respect to the canonical measure $\pi_{u, v}^{2}$ by

$$
\Phi(u, v):=\mathbf{E}_{u, v}(\phi), \quad \Psi(u, v):=\mathbf{E}_{u, v}(\psi) .
$$

The following lemma was proved in [15].

Lemma 1. Suppose we have a particle system with two conserved quantities and rates satisfying conditions (A) and $(\mathrm{C})$. Then

$$
\partial_{\theta} \Psi(u(\theta, \tau), v(\theta, \tau))=\partial_{\tau} \Phi(u(\theta, \tau), v(\theta, \tau)) .
$$

The first derivative matrix of the fluxes $\Phi$ and $\Psi$ (with resp. to $u, v$ ) will be denoted by

$$
D=D(u, v):=\left(\begin{array}{ll}
\Phi_{u} & \Phi_{v} \\
\Psi_{u} & \Psi_{v}
\end{array}\right)
$$

From Lemma 1 it follows that $D(u, v)$ is (weakly) hyperbolic, it can be diagonalized in a real sense (see [15]). We denote the two eigenvalues of $D$ by $\lambda$ and $\mu$, and the corresponding right and left eigenvectors by $\mathbf{r}=\left(r_{1}, r_{2}\right)^{\dagger}, \mathbf{s}=$ $\left(s_{1}, s_{2}\right)^{\dagger}$ and $\mathbf{l}=\left(l_{1}, l_{2}\right), \mathbf{m}=\left(m_{1}, m_{2}\right)$ :

$$
\begin{array}{ll}
D \mathbf{r}=\lambda \mathbf{r}, & \mathbf{l} D=\lambda \mathbf{l}, \\
D \mathbf{s}=\mu \mathbf{s}, & \mathbf{m} D=\mu \mathbf{m} .
\end{array}
$$

Although we do not denote it explicitly, all of these are functions of $(u, v)$. We can assume

$$
|\mathbf{r}|=|\mathbf{s}|=1, \quad \mathbf{l} \cdot \mathbf{r}=1, \quad \mathbf{m} \cdot \mathbf{s}=1 .
$$

The second derivatives of the macroscopic fluxes are denoted by $\Phi^{\prime \prime}, \Psi^{\prime \prime}$, these are symmetric two-by-two matrices depending on $(u, v)$.

\subsection{The spectral gap condition}

Let $l$ be a positive integer and $Z, N$ be integers with $Z \in[l \min \zeta, l \max \zeta], N \in[l \min \eta, l \max \eta]$. Expectation with respect to the measure $\pi_{Z, N}^{l}$ is denoted by $\mathbf{E}_{Z, N}^{l}(\cdot)$. For $f: \Omega_{Z, N}^{l} \rightarrow \mathbb{R}$ let 


$$
\begin{aligned}
& L_{Z, N}^{l} f(\underline{\omega}):=\sum_{j=1}^{l-1} \sum_{\omega^{\prime}, \omega^{\prime \prime}} r\left(\omega_{j}, \omega_{j+1} ; \omega^{\prime}, \omega^{\prime \prime}\right)\left(f\left(\Theta_{j, j+1}^{\omega^{\prime}, \omega^{\prime \prime}} \underline{\omega}\right)-f(\underline{\omega})\right), \\
& D_{Z, N}^{l}(f):=\frac{1}{2} \sum_{j=1}^{l-1} \mathbf{E}_{Z, N}^{l}\left(\sum_{\omega^{\prime}, \omega^{\prime \prime}} r\left(\omega_{j}, \omega_{j+1} ; \omega^{\prime}, \omega^{\prime \prime}\right)\left(f\left(\Theta_{j, j+1}^{\omega^{\prime}, \omega^{\prime \prime}}\right)-f(\underline{\omega})\right)^{2}\right) .
\end{aligned}
$$

$L_{Z, N}^{l}$ is the infinitesimal generator restricted to the hyperplane $\Omega_{Z, N}^{l}$, and $D_{Z, N}^{l}$ is the Dirichlet form associated to $L_{Z, N}^{l}$ (or to its symmetric part). Note, that $L_{Z, N}^{l}$ is defined with free boundary conditions.

We will assume the following additional condition on our models:

(D) There exists a positive constant $W$ independent of $l, Z, N$ such that for any $f: \Omega_{Z, N}^{l} \rightarrow \mathbb{R}$ with $\mathbf{E}_{Z, N}^{l} f=0$, the following bound holds:

$$
\mathbf{E}_{N, Z}^{l} f^{2} \leqslant W l^{2} D_{Z, N}^{l}(f)
$$

\section{Remarks.}

1. Presumably (D) is true for all (or a large class of) the models satisfying conditions (A)-(C). The techniques of [9] should be suitable to get the desired gap estimates, but we do not know about any published results covering the general case.

2. It is hoped (but not proved in a general setting) that the following stronger condition also holds for a wide range of models in our framework. $\left(\mathrm{D}^{\prime}\right)$ is called the logarithmic-Sobolev inequality and it implies (D).

$\left(\mathrm{D}^{\prime}\right)$ There exists a positive constant $W$ independent of $l, Z, N$ such that for any $f: \Omega_{Z, N}^{l} \rightarrow \mathbb{R}_{+}$with $\mathbf{E}_{Z, N}^{l} f=1$, the following bound holds:

$$
\mathbf{E}_{N, Z}^{l} f \log f \leqslant W l^{2} D_{Z, N}^{l}(\sqrt{f}) .
$$

Actually, our specific examples all satisfy $\left(\mathrm{D}^{\prime}\right)$, but we state the theorem assuming only (D) to make it less restrictive.

\subsection{Examples}

$\{-1,0,+1\}$-model. The model is described and analyzed in full detail in [6] and [15]. The one spin state space is $\Omega=\{-1,0,+1\}$. The dynamics consists of nearest neighbor spin exchanges and the two conserved quantities are $\zeta(\omega)=\omega$ and $\eta(\omega)=1-|\omega|$. The jump rates are

$$
\begin{array}{ll}
r(1,-1 ;-1,1)=0, & r(-1,1 ; 1,-1)=2, \\
r(0,-1 ;-1,0)=0, & r(-1,0 ; 0,-1)=1, \\
r(1,0 ; 0,1)=0, & r(0,1 ; 1,0)=1 .
\end{array}
$$

The one-dimensional marginals of the stationary measures are

$$
\pi_{u, v}(0)=v, \quad \pi_{u, v}( \pm 1)=\frac{1-v \pm u}{2}
$$

with the domain of variables $\mathcal{D}=\left\{(u, v) \in \mathbb{R}_{+} \times \mathbb{R}:|u|+v \leqslant 1\right\}$. The macroscopic fluxes are $\Phi(u, v)=u^{2}+v$, $\Psi(u, v)=u v$. In [6] it was shown that the log-Sobolev bound holds for this model. We also remark that in that paper the Eulerian hydrodynamic limit is proved for the model, even after the appearance of shocks. 
Two-lane models. Let $\Omega=\{0,1, \ldots, \bar{n}\} \times\{-\bar{z},-\bar{z}+1, \ldots, \bar{z}-1, \bar{z}\}$, where $\bar{n} \in \mathbb{N}$ and $\bar{z} \in\left\{\frac{1}{2}, 1, \frac{3}{2}, 2, \ldots\right\}$. The elements of $\Omega$ will be denoted $\omega:=\left(\begin{array}{c}\eta \\ \zeta\end{array}\right), \sum_{j} \eta_{j}$ and $\sum_{j} \zeta_{j}$ will be the conserved quantities of the dynamics. We allow only the following elementary changes to occur at neighboring sites $j, j+1$ :

$$
\left(\begin{array}{cc}
\eta_{j} & \eta_{j+1} \\
\zeta_{j} & \zeta_{j+1}
\end{array}\right) \rightarrow\left(\begin{array}{cc}
\eta_{j} & \eta_{j+1} \\
\zeta_{j} \mp 1 & \zeta_{j+1} \pm 1
\end{array}\right), \quad\left(\begin{array}{cc}
\eta_{j} & \eta_{j+1} \\
\zeta_{j} & \zeta_{j+1}
\end{array}\right) \rightarrow\left(\begin{array}{cc}
\eta_{j} \mp 1 & \eta_{j+1} \pm 1 \\
\zeta_{j} & \zeta_{j+1}
\end{array}\right)
$$

with appropriate rates. Beside the conditions already imposed on we also assume that the one-dimensional marginals of the steady state measures factorize as follows:

$$
\pi(\omega)=\pi\left(\begin{array}{l}
\eta \\
\zeta
\end{array}\right)=p(\eta) q(\zeta)
$$

The simplest case, with $\bar{n}=1$ and $\bar{z}=1 / 2$, that is with $\Omega=\{0,1\} \times\{-1 / 2,+1 / 2\}$, was introduced and fully analyzed in [11] and [15]. In general the conditions (A)-(C) impose some nontrivial combinatorial constraints on the rates which are satisfied by a finite parameter family of models (the number of free parameters increases with $\bar{n}$ and $\bar{z}$ ).

In [17] the logarithmic Sobolev inequality was proved for symmetric $K$-exclusion processes. Because of the product structure (10) of the invariant measure this also implies that it also holds for the two-lane models, condition $\left(\mathrm{D}^{\prime}\right)$ is satisfied.

\section{Perturbation of the Eulerian hdl}

In [15] it was proved by the application of Yau's relative entropy method, that under Eulerian scaling the local density profiles of the conserved quantities evolve according to the following system of partial differential equations:

$$
\left\{\begin{array}{l}
\partial_{t} u+\partial_{x} \Phi(u, v)=0 \\
\partial_{t} v+\partial_{x} \Psi(u, v)=0 .
\end{array}\right.
$$

This pde is usually a strictly hyperbolic conservation law (i.e. $D(u, v)$ has two distinct real eigenvalues), weak hyperbolicity follows from Lemma 1 (see [15]). Since the relative entropy method needs smoothness conditions for the solution of the limiting equation, the previous result holds only up to a finite time, till the appearance of the first shock.

\subsection{Formal perturbation}

We will investigate the hydrodynamic behavior of small perturbations of an equilibrium point. For that we need to understand the asymptotics of small perturbations of a constant solution of (11). One of the perturbation techniques is the so-called method of weakly nonlinear geometric optics (see e.g. [4,7]) which gives the following formal result.

Fix a point $\left(u_{0}, v_{0}\right)$ in $\mathcal{D}$ and suppose that this point is strictly hyperbolic, i.e.

$$
\lambda \neq \mu
$$

at $\left(u_{0}, v_{0}\right)$. Suppose $\left(u_{\varepsilon}(t, x), v_{\varepsilon}(t, x)\right)$ is the solution of the pde (11) with initial conditions

$$
\begin{aligned}
& u_{\varepsilon}(0, x)=u_{0}+\varepsilon u^{*}(x), \\
& v_{\varepsilon}(0, x)=v_{0}+\varepsilon v^{*}(x),
\end{aligned}
$$


where $u^{*}(x), v^{*}(x)$ are fixed $\mathbb{T} \mapsto \mathbb{R}$ smooth functions. Denote

$$
\begin{array}{ll}
\sigma_{0}(x):=\mathbf{l} \cdot\left(u^{*}(x), v^{*}(x)\right)^{\dagger}, & c_{\sigma}:=\int_{\mathbb{T}} \sigma_{0}(y) \mathrm{d} y, \\
\delta_{0}(x):=\mathbf{m} \cdot\left(u^{*}(x), v^{*}(x)\right)^{\dagger}, & c_{\delta}:=\int_{\mathbb{T}} \delta_{0}(y) \mathrm{d} y,
\end{array}
$$

and

$$
\begin{array}{ll}
a_{1}:=\mathbf{l} \cdot\left(\mathbf{r}^{\dagger} \Phi^{\prime \prime} \mathbf{r}, \mathbf{r}^{\dagger} \Psi^{\prime \prime} \mathbf{r}\right)^{\dagger}, & a_{2}:=\mathbf{l} \cdot\left(\mathbf{r}^{\dagger} \Phi^{\prime \prime} \mathbf{s}, \mathbf{r}^{\dagger} \Psi^{\prime \prime} \mathbf{s}\right)^{\dagger}, \\
b_{1}:=\mathbf{m} \cdot\left(\mathbf{s}^{\dagger} \Phi^{\prime \prime} \mathbf{s}, \mathbf{s}^{\dagger} \Psi^{\prime \prime} \mathbf{s}\right)^{\dagger}, & b_{2}:=\mathbf{m} \cdot\left(\mathbf{r}^{\dagger} \Phi^{\prime \prime} \mathbf{s}, \mathbf{r}^{\dagger} \Psi^{\prime \prime} \mathbf{s}\right)^{\dagger},
\end{array}
$$

where $\mathbf{l}, \mathbf{m}, \mathbf{r}, \mathbf{s}$ and $\Phi^{\prime \prime}, \Psi^{\prime \prime}$ are the respective vector- and matrix-valued functions taken at $\left(u_{0}, v_{0}\right)$.

Then, according to the formal computations of the geometric optics method,

$$
\left(\begin{array}{c}
u_{\varepsilon}(t, x) \\
v_{\varepsilon}(t, x)
\end{array}\right)=\left(\begin{array}{c}
u_{0} \\
v_{0}
\end{array}\right)+\varepsilon \sigma(\varepsilon t, x-\lambda t)\left(\begin{array}{l}
r_{1} \\
r_{2}
\end{array}\right)+\varepsilon \delta(\varepsilon t, x-\mu t)\left(\begin{array}{l}
s_{1} \\
s_{2}
\end{array}\right)+\mathcal{O}\left(\varepsilon^{2}\right),
$$

as $\varepsilon \rightarrow 0$, where $\sigma$ and $\delta$ are the solutions of the following Cauchy problems:

$$
\left\{\begin{array}{l}
\partial_{t} \sigma(t, x)+\partial_{x}\left(a_{1} \cdot \frac{1}{2} \sigma(t, x)^{2}+c_{\delta} a_{2} \sigma(t, x)\right)=0, \\
\sigma(0, x)=\sigma_{0}(x)
\end{array}\right.
$$

and

$$
\left\{\begin{array}{l}
\partial_{t} \delta(t, x)+\partial_{x}\left(b_{1} \cdot \frac{1}{2} \delta(t, x)^{2}+c_{\sigma} b_{2} \delta(t, x)\right)=0 \\
\delta(0, x)=\delta_{0}(x)
\end{array}\right.
$$

\section{Remarks.}

1. This result means that a small perturbation of a constant solution of (11) is governed by the solutions of two decoupled equations (at least, by formal computations). If $a_{1}$ and $b_{1}$ are nonzero, then these equations are linear transforms of the Burgers equation. Otherwise the respective equations become linear transport equations. It is easy to check, that $a_{1} \neq 0, b_{1} \neq 0$ hold exactly when the point $\left(u_{0}, v_{0}\right)$ is genuinely nonlinear, i.e.

$$
\nabla \lambda \cdot \mathbf{r} \neq 0, \quad \nabla \mu \cdot \mathbf{s} \neq 0
$$

at $\left(u_{0}, v_{0}\right)$.

2. The geometric optics method is based on series expansion, thus it needs smoothness as a condition which could only be true up to a finite time in our case. Surprisingly, this formal method gives good approximation of the solutions even after the shocks. In [4] the authors prove that Eq. (15) is valid, in the sense that for any $t>0$ the $\mathrm{L}_{1}$-norm of the difference of the two sides is bounded by $C t \varepsilon^{2}$. In fact, this result is valid for the case if we consider the pde (11) on $\mathbb{T}$ (as we do), on $\mathbb{R}$ they have even stronger bounds.

\subsection{The main result}

Our main theorem gives a similar result on the microscopic level. We will apply Yau's method, thus our results will hold in the regime of smooth solutions.

Suppose, that $\left(u_{0}, v_{0}\right)$ is a point inside the physical domain which is strictly hyperbolic, see (12). Let $u^{*}(x), v^{*}(x): \mathbb{T} \rightarrow \mathbb{R}$ be smooth functions. Define $\sigma(t, x), \delta(t, x)$ according to (13), (14), (16) and (17), and suppose that they are smooth in $\mathbb{T} \times[0, T]$. Fix a small positive parameter $\beta$, and suppose that a particle system on $\Omega^{n}$ satisfying conditions (A)-(D) has initial distribution for which the density profiles of the two conserved quantities are 'close' to the functions $u_{0}+n^{-\beta} u^{*}(\cdot), v_{0}+n^{-\beta} v^{*}(\cdot)$. I.e. the profiles are a small perturbation of the constant $\left(u_{0}, v_{0}\right)$ profile. Clearly, $\left(u_{0}+n^{-\beta} u^{*}(x), v_{0}+n^{-\beta} v^{*}(x)\right) \in \mathcal{D}$ holds for every $x \in \mathbb{T}$, for $n$ large 
enough. Then, uniformly for $0 \leqslant t \leqslant T$, at time $n^{1+\beta} t$ the respective density profiles will be 'close' to the functions $u_{0}+n^{-\beta} u^{(n)}(t, \cdot), v_{0}+n^{-\beta} v^{(n)}(t, \cdot)$, where

$$
\left(\begin{array}{c}
u^{(n)}(t, x) \\
v^{(n)}(t, x)
\end{array}\right):=\sigma\left(t, x-\lambda n^{\beta} t\right)\left(\begin{array}{l}
r_{1} \\
r_{2}
\end{array}\right)+\delta\left(t, x-\mu n^{\beta} t\right)\left(\begin{array}{l}
s_{1} \\
s_{2}
\end{array}\right) .
$$

For the precise formulation of the result we need to introduce some additional notations. We will denote by $\mu_{t}^{n}$ the true distribution of the system at microscopic time $n^{1+\beta} t$ :

$$
\mu_{t}^{n}:=\mu_{0}^{n} \exp \left\{n^{1+\beta} t L^{n}\right\} .
$$

We define the time-dependent reference measure $v_{t}^{n}$ as

$$
v_{t}^{n}:=\prod_{j \in \mathbb{T}^{n}} \pi_{u_{0}+n^{-\beta} u^{(n)}(t, j / n), v_{0}+n^{-\beta} v^{(n)}(t, j / n)},
$$

with $u^{(n)}, v^{(n)}$ defined in (18). This measure mimics on a microscopic level the macroscopic profiles $u_{0}+$ $n^{-\beta} u^{(n)}(t, \cdot), v_{0}+n^{-\beta} v^{(n)}(t, \cdot)$. We also choose an absolute reference measure

$$
\pi^{n}:=\prod_{j \in \mathbb{T}^{n}} \pi_{u_{0}, v_{0}},
$$

which is a stationary measure of our Markov process on $\Omega^{n}$.

Theorem. Let $\beta \in\left(0, \frac{1}{5}\right)$ be fixed. Under the stated conditions, if

$$
H\left(\mu_{0}^{n} \mid v_{0}^{n}\right)=\mathrm{o}\left(n^{1-2 \beta}\right),
$$

then

$$
H\left(\mu_{t}^{n} \mid v_{t}^{n}\right)=\mathrm{o}\left(n^{1-2 \beta}\right),
$$

uniformly for $0 \leqslant t \leqslant T$.

The following corollary is a simple consequence of the theorem and the entropy inequality.

Corollary. Assume the conditions of the Theorem. Let $g: \mathbb{T} \rightarrow \mathbb{R}$ be a test function. Then for any $t \in[0, T]$

$$
\begin{aligned}
& \left|n^{-1+\beta} \sum_{j \in \mathbb{T}^{n}} g\left(\frac{j}{n}\right)\left(\zeta_{j}\left(n^{1+\beta} t\right)-u_{0}\right)-\int_{\mathbb{T}} g(x)\left(\sigma\left(t, x-\lambda n^{\beta} t\right) r_{1}+\delta\left(t, x-\mu n^{\beta} t\right) s_{1}\right) \mathrm{d} x\right| \stackrel{\mathbf{P}}{\longrightarrow} 0, \\
& \left|n^{-1+\beta} \sum_{j \in \mathbb{T}^{n}} g\left(\frac{j}{n}\right)\left(\eta_{j}\left(n^{1+\beta} t\right)-v_{0}\right)-\int_{\mathbb{T}} g(x)\left(\sigma\left(t, x-\lambda n^{\beta} t\right) r_{2}+\delta\left(t, x-\mu n^{\beta} t\right) s_{2}\right) \mathrm{d} x\right| \stackrel{\mathbf{P}}{\longrightarrow} 0 .
\end{aligned}
$$

\section{Remarks.}

1. The theorem states that if the initial distribution of the system is 'close' to $v_{0}^{n}$ in relative entropy sense then at time $n^{1+\beta} t$ it will be close to $v_{t}^{n}$. The fact, that 'close' should mean o $\left(n^{1-2 \beta}\right)$ can be easily justified, see e.g. [14] or [15].

2. If instead of condition (D) we assume the log-Sobolev bound $\left(\mathrm{D}^{\prime}\right)$ then our theorem is valid for $\beta \in\left(0, \frac{1}{3}\right)$.

3. A similar result holds if the point $\left(u_{0}, v_{0}\right)$ is on the boundary of $\mathcal{D}$. In that case on the right sides of Eqs. (22), (23) we have $\mathrm{o}\left(n^{1-\beta}\right)$ instead of $\mathrm{o}\left(n^{1-2 \beta}\right)$. The proof is essentially the same, although some minor modifications are needed. 


\section{Proof}

We will assume, that

$$
\left(u_{0}, v_{0}\right)=(0,0), \quad \Phi_{v}(0,0)=\Psi_{u}(0,0)=0 .
$$

It is easy to see, that we can always reduce the general case to get (24), via some suitable linear transformations on $(\zeta, \eta)$. Also, we may assume

$$
\theta(0,0)=\tau(0,0)=0
$$

and with the proper choice of the constants in the definition (7) we can set

$$
\Phi(0,0)=\Psi(0,0)=0 .
$$

Assumptions (24) imply, that

$$
D=\left(\begin{array}{cc}
\lambda & 0 \\
0 & \mu
\end{array}\right), \quad \mathbf{l}=\mathbf{r}^{\dagger}=(1,0), \quad \mathbf{m}=\mathbf{s}^{\dagger}=(0,1),
$$

and

$$
u^{(n)}(t, x)=\sigma\left(t, x-\lambda n^{\beta} t\right), \quad v^{(n)}(t, x)=\delta\left(t, x-\mu n^{\beta} t\right) .
$$

We introduce the notations

$$
\Phi^{\prime \prime}=\left(\begin{array}{ll}
\Phi_{u u} & \Phi_{u v} \\
\Phi_{v u} & \Phi_{v v}
\end{array}\right)=:\left(\begin{array}{ll}
a_{1} & a_{2} \\
a_{2} & a_{3}
\end{array}\right), \quad \Psi^{\prime \prime}=\left(\begin{array}{ll}
\Psi_{u u} & \Psi_{u v} \\
\Psi_{v u} & \Psi_{v v}
\end{array}\right)=:\left(\begin{array}{ll}
b_{3} & b_{2} \\
b_{2} & b_{1}
\end{array}\right) .
$$

Clearly, these definitions agree with the definition (14) of $a_{1}, a_{2}, b_{1}, b_{2}$.

We define the functions $\bar{\sigma}\left(t, x_{1}, x_{2}\right), \bar{\delta}\left(t, x_{1}, x_{2}\right)$ as

$$
\begin{aligned}
& \bar{\sigma}\left(t, x_{1}, x_{2}\right):=\frac{1}{\mu-\lambda}\left(a_{2} \sigma\left(t, x_{1}\right) \delta\left(t, x_{2}\right)+a_{2} \sigma_{x}\left(t, x_{1}\right) \int_{0}^{x_{2}}\left(\delta(t, z)-c_{\delta}\right) \mathrm{d} z+\frac{a_{3}}{2} \delta\left(t, x_{2}\right)^{2}\right), \\
& \bar{\delta}\left(t, x_{1}, x_{2}\right):=\frac{1}{\lambda-\mu}\left(b_{2} \sigma\left(t, x_{1}\right) \delta\left(t, x_{2}\right)+b_{2} \delta_{x}\left(t, x_{2}\right) \int_{0}^{x_{1}}\left(\sigma(t, z)-c_{\sigma}\right) \mathrm{d} z+\frac{b_{3}}{2} \sigma\left(t, x_{1}\right)^{2}\right)
\end{aligned}
$$

(see (13) for the definitions of $c_{\sigma}, c_{\delta}$ ). The defining partial differential equations (16), (17) of the functions $\sigma, \delta$ are conservation laws, thus for any $0 \leqslant t \leqslant T$ :

$$
\int_{\mathbb{T}} \sigma(t, z) \mathrm{d} z=c_{\sigma}, \quad \int_{\mathbb{T}} \delta(t, z) \mathrm{d} z=c_{\delta} .
$$

From that it follows that $\bar{\sigma}, \bar{\delta}$ are well-defined smooth functions on $[0, T] \times \mathbb{T} \times \mathbb{T}$ (i.e. periodic in $x_{1}$ and $x_{2}$ ) with bounded derivatives.

\subsection{Changing the time-dependent reference measure}

The usual way to prove a result like the Theorem is to get a Grönwall-type estimate on $H\left(\mu_{t}^{n} \mid v_{t}^{n}\right)$ :

$$
H\left(\mu_{t}^{n} \mid \nu_{t}^{n}\right)-H\left(\mu_{0}^{n} \mid \nu_{0}^{n}\right) \leqslant C \int_{0}^{t} H\left(\mu_{s}^{n} \mid \nu_{s}^{n}\right) \mathrm{d} s+\mathrm{o}\left(n^{1-2 \beta}\right),
$$


via bounding the derivative $\partial_{t} H\left(\mu_{t}^{n} \mid v_{t}^{n}\right)$. We will use a slightly different approach, by proving a similar estimate for $H\left(\mu_{t}^{n} \mid \tilde{v}_{t}^{n}\right)$ :

$$
H\left(\mu_{t}^{n} \mid \tilde{v}_{t}^{n}\right)-H\left(\mu_{0}^{n} \mid \tilde{v}_{0}^{n}\right) \leqslant C \int_{0}^{t} H\left(\mu_{s}^{n} \mid \tilde{v}_{s}^{n}\right) \mathrm{d} s+\mathrm{o}\left(n^{1-2 \beta}\right) .
$$

Here

$$
\tilde{v}_{t}^{n}:=\prod_{j \in \mathbb{T}^{n}} \pi_{n^{-\beta} \tilde{u}^{(n)}(t, j / n), n^{-\beta} \tilde{v}^{(n)}(t, j / n)}
$$

and $\tilde{u}^{(n)}, \tilde{v}^{(n)}$ are smooth functions defined as

$$
\begin{aligned}
\tilde{u}^{(n)}(t, x) & :=u^{(n)}(t, x)+n^{-\beta} \bar{\sigma}\left(t, x-\lambda n^{\beta} t, x-\mu n^{\beta} t\right) \\
& =\sigma\left(t, x-\lambda n^{\beta} t\right)+n^{-\beta} \bar{\sigma}\left(t, x-\lambda n^{\beta} t, x-\mu n^{\beta} t\right), \\
\tilde{v}^{(n)}(t, x) & :=v^{(n)}(t, x)+n^{-\beta} \bar{\delta}\left(t, x-\lambda n^{\beta} t, x-\mu n^{\beta} t\right), \\
& =\delta\left(t, x-\mu n^{\beta} t\right)+n^{-\beta} \bar{\delta}\left(t, x-\lambda n^{\beta} t, x-\mu n^{\beta} t\right) .
\end{aligned}
$$

Because of Lemma 2 below and condition (22) we have $H\left(\mu_{0}^{n} \mid \tilde{v}_{0}^{n}\right)=\mathrm{o}\left(n^{1-2 \beta}\right)$. Thus from (31)

$$
H\left(\mu_{t}^{n} \mid \tilde{v}_{t}^{n}\right)=\mathrm{o}\left(n^{1-2 \beta}\right)
$$

will follow uniformly for $0 \leqslant t \leqslant T$. Using Lemma 2 again we get the Theorem.

Lemma 2. Let $\mu_{t}^{n}, v_{t}^{n}, \tilde{v}_{t}^{n}$ be the measures defined as before, with $t \in[0, T]$. Then

$$
H\left(\mu_{t}^{n} \mid v_{t}^{n}\right)=\mathrm{o}\left(n^{1-2 \beta}\right) \Longleftrightarrow H\left(\mu_{t}^{n} \mid \tilde{v}_{t}^{n}\right)=\mathrm{o}\left(n^{1-2 \beta}\right) .
$$

Proof. We start with

$$
H\left(\mu_{t}^{n} \mid v_{t}^{n}\right)-H\left(\mu_{t}^{n} \mid \tilde{v}_{t}^{n}\right)=-\int_{\Omega^{n}} \log \frac{\mathrm{d} v_{t}^{n}}{\mathrm{~d} \tilde{v}_{t}^{n}} \mathrm{~d} \mu_{t}^{n} .
$$

By Subsections 2.2 and 2.3 we can calculate that

$$
\begin{aligned}
\log \frac{\mathrm{d} v_{t}^{n}}{\mathrm{~d} \tilde{v}_{t}^{n}}(\underline{\omega})= & \sum_{j \in \mathbb{T}^{n}}\left\{\left(\theta\left(n^{-\beta} u^{(n)}, n^{-\beta} v^{(n)}\right)-\theta\left(n^{-\beta} \tilde{u}^{(n)}, n^{-\beta} \tilde{v}^{(n)}\right)\right) \zeta_{j}\right. \\
& +\left(\tau\left(n^{-\beta} u^{(n)}, n^{-\beta} v^{(n)}\right)-\tau\left(n^{-\beta} \tilde{u}^{(n)}, n^{-\beta} \tilde{v}^{(n)}\right)\right) \eta_{j} \\
& -G\left(\theta\left(n^{-\beta} u^{(n)}, n^{-\beta} v^{(n)}\right), \tau\left(n^{-\beta} u^{(n)}, n^{-\beta} v^{(n)}\right)\right) \\
& \left.+G\left(\theta\left(n^{-\beta} \tilde{u}^{(n)}, n^{-\beta} \tilde{v}^{(n)}\right), \tau\left(n^{-\beta} \tilde{u}^{(n)}, n^{-\beta} \tilde{v}^{(n)}\right)\right)\right\},
\end{aligned}
$$

where, for typographical reasons, we omitted the arguments $\left(t, \frac{j}{n}\right)$ from the functions $u^{(n)}, v^{(n)}, \tilde{u}^{(n)}, \tilde{v}^{(n)}$.

From the previous expression via power-series expansion:

$$
\begin{aligned}
\left|\log \frac{\mathrm{d} v_{t}^{n}}{\mathrm{~d} \tilde{v}_{t}^{n}}(\underline{\omega})\right| & \leqslant \mathcal{O}\left(n^{1-3 \beta}\right)+C n^{-2 \beta} \sum_{j \in \mathbb{T}^{n}}\left(\left|\zeta_{j}-u^{(n)}\left(t, \frac{j}{n}\right)\right|+\left|\eta_{j}-v^{(n)}\left(t, \frac{j}{n}\right)\right|\right) \\
& =\mathcal{O}\left(n^{1-3 \beta}\right)+C n^{-2 \beta} \sum_{j \in \mathbb{T}^{n}}\left(\left|\zeta_{j}-\tilde{u}^{(n)}\left(t, \frac{j}{n}\right)\right|+\left|\eta_{j}-\tilde{v}^{(n)}\left(t, \frac{j}{n}\right)\right|\right),
\end{aligned}
$$


with uniform error terms. Using this with (34) and the entropy inequality with respect to $v_{t}^{n}$ and $\tilde{v}_{t}^{n}$ the lemma follows.

We also note, that applying the same arguments as in the proof of Lemma 2 we get

$$
H\left(\mu_{0}^{n} \mid \pi^{n}\right)=\mathcal{O}\left(n^{1-2 \beta}\right)
$$

from condition (22). Since $\pi^{n}$ is a stationary measure,

$$
H\left(\mu_{t}^{n} \mid \pi^{n}\right) \leqslant H\left(\mu_{0}^{n} \mid \pi^{n}\right)=\mathcal{O}\left(n^{1-2 \beta}\right)
$$

for all $t \geqslant 0$.

The proof of the following lemma is a simple application of the entropy inequality with the entropy bound (35). Mind that because of (24) and (26) we have

$$
\int_{\Omega^{n}} \zeta_{i} \mathrm{~d} \pi^{n}=\int_{\Omega^{n}} \eta_{i} \mathrm{~d} \pi^{n}=\int_{\Omega^{n}} \phi_{i} \mathrm{~d} \pi^{n}=\int_{\Omega^{n}} \psi_{i} \mathrm{~d} \pi^{n}=0 .
$$

Lemma 3. Suppose $b_{1}, b_{2}, \ldots$ are real numbers with $\left|b_{j}\right| \leqslant 1$ and $\xi_{j}$ stands for either $\eta_{j}, \zeta_{j}, \psi_{j}$ or $\phi_{j}$. Then

$$
\int_{\Omega^{n}} \frac{1}{n} \sum_{j \in \mathbb{T}^{n}} b_{j} \xi_{j} \mathrm{~d} \mu_{t}^{n} \leqslant C n^{-\beta}
$$

with an absolute constant $C$.

In the rest of the paper we prove the inequality (31).

\subsection{Preparatory computations}

We define

$$
\begin{aligned}
& \tilde{\theta}^{(n)}(t, x):=n^{\beta} \theta\left(n^{-\beta} \tilde{u}^{(n)}(t, x), n^{-\beta} \tilde{v}^{(n)}(t, x)\right), \\
& \tilde{\tau}^{(n)}(t, x):=n^{\beta} \tau\left(n^{-\beta} \tilde{u}^{(n)}(t, x), n^{-\beta} \tilde{v}^{(n)}(t, x)\right) .
\end{aligned}
$$

It is easy to check, that the partial derivatives $\partial_{x} \tilde{\theta}^{(n)}(t, x), \partial_{x} \tilde{\tau}^{(n)}(t, x), \partial_{x}^{2} \tilde{\theta}^{(n)}(t, x), \partial_{x}^{2} \tilde{\tau}^{(n)}(t, x)$ are uniformly bounded in $[0, T] \times \mathbb{T}$. We will exploit this fact several times during the proof, mostly without mentioning it. From Subsection 2.2 we have

$$
\begin{aligned}
\tilde{f}_{t}^{n}:=\frac{\mathrm{d} \tilde{\nu}_{t}^{n}}{\mathrm{~d} \pi^{n}}= & \exp \sum_{j \in \mathbb{T}^{n}}\left\{n^{-\beta}\left(\tilde{\theta}^{(n)}\left(t, \frac{j}{n}\right)-\theta_{0}^{n}\right) \zeta_{j}+n^{-\beta}\left(\tilde{\tau}^{(n)}\left(t, \frac{j}{n}\right)-\tau_{0}^{n}\right) \eta_{j}\right. \\
& \left.-G\left(n^{-\beta} \tilde{\theta}^{(n)}\left(t, \frac{j}{n}\right), n^{-\beta} \tilde{\tau}^{(n)}\left(t, \frac{j}{n}\right)\right)\right\} .
\end{aligned}
$$

Differentiating the identity

$$
H\left(\mu_{t}^{n} \mid \tilde{v}_{t}^{n}\right)-H\left(\mu_{t}^{n} \mid \pi^{n}\right)=-\int_{\Omega^{n}} \log \tilde{f}_{t}^{n} \mathrm{~d} \mu_{t}^{n}
$$

and noting that $\partial_{t} H\left(\mu_{t}^{n} \mid \pi^{n}\right) \leqslant 0$ we get the following bound on $\partial_{t} H\left(\mu_{t}^{n} \mid \tilde{v}_{t}^{n}\right)$ :

$$
n^{2 \beta-1} \partial_{t} H\left(\mu_{t}^{n} \mid \tilde{v}_{t}^{n}\right) \leqslant-\int_{\Omega^{n}}\left(n^{3 \beta} L^{n} \log \tilde{f}_{t}^{n}+n^{-1+2 \beta} \partial_{t} \log \tilde{f}_{t}^{n}\right) \mathrm{d} \mu_{t}^{n} .
$$


Integrating with respect to the time:

$$
n^{2 \beta-1}\left(H\left(\mu_{t}^{n} \mid \tilde{v}_{t}^{n}\right)-H\left(\mu_{0}^{n} \mid \tilde{v}_{0}^{n}\right)\right) \leqslant-\int_{0}^{t} \int_{\Omega^{n}}\left(n^{3 \beta} L^{n} \log \tilde{f}_{s}^{n}+n^{-1+2 \beta} \partial_{t} \log \tilde{f}_{s}^{n}\right) \mathrm{d} \mu_{s}^{n} \mathrm{~d} s .
$$

We estimate the two terms on the right-hand side separately in the next two subsections.

\subsection{Estimating the first term of (38)}

From the definitions

$$
\begin{aligned}
n^{3 \beta} L^{n} \log \tilde{f}_{t}^{n}(\underline{\omega})= & n^{-1+2 \beta} \sum_{j \in \mathbb{T}^{n}}\left(\phi_{j}-\Phi\left(n^{-\beta} \tilde{u}_{j}^{(n)}, n^{-\beta} \tilde{v}_{j}^{(n)}\right)\right) \partial_{x} \tilde{\theta}^{(n)}\left(t, \frac{j}{n}\right) \\
& +\left(\psi_{j}-\Psi\left(n^{-\beta} \tilde{u}_{j}^{(n)}, n^{-\beta} \tilde{v}_{j}^{(n)}\right)\right) \partial_{x} \tilde{\tau}^{(n)}\left(t, \frac{j}{n}\right)+\operatorname{Err}_{1}^{n}(t, \underline{\omega})+\operatorname{Err}_{2}^{n}(t),
\end{aligned}
$$

where

$$
\begin{aligned}
\operatorname{Err}_{1}^{n}(t, \underline{\omega}):= & n^{-1+2 \beta} \sum_{j \in \mathbb{T}^{n}}\left\{\phi_{j}\left(\nabla^{n} \tilde{\theta}^{(n)}\left(t, \frac{j}{n}\right)-\partial_{x} \tilde{\theta}^{(n)}\left(t, \frac{j}{n}\right)\right)\right. \\
& \left.+\psi_{j}\left(\nabla^{n} \tilde{\tau}^{(n)}\left(t, \frac{j}{n}\right)-\partial_{x} \tilde{\tau}^{(n)}\left(t, \frac{j}{n}\right)\right)\right\}, \\
\operatorname{Err}_{2}^{n}(t):= & n^{-1+2 \beta} \sum_{j \in \mathbb{T}^{n}}\left\{\Phi\left(n^{-\beta} \tilde{u}_{j}^{(n)}, n^{-\beta} \tilde{v}_{j}^{(n)}\right) \partial_{x} \tilde{\theta}^{(n)}\left(t, \frac{j}{n}\right)\right. \\
+ & \left.\Psi\left(n^{-\beta} \tilde{u}_{j}^{(n)}, n^{-\beta} \tilde{v}_{j}^{(n)}\right) \partial_{x} \tilde{\tau}^{(n)}\left(t, \frac{j}{n}\right)\right\} .
\end{aligned}
$$

We used the (slightly abused) shorthanded notations

$$
\tilde{u}_{j}^{(n)}=\tilde{u}^{(n)}\left(t, \frac{j}{n}\right), \quad \tilde{v}_{j}^{(n)}=\tilde{v}^{(n)}\left(t, \frac{j}{n}\right),
$$

and $\nabla^{n}$ denotes the discrete gradient:

$$
\nabla^{n} g(x):=n\left(g\left(x+\frac{1}{n}\right)-g(x)\right)
$$

Using the smoothness of $\tilde{\theta}^{(n)}, \tilde{\tau}^{(n)}$ and Lemma 3 the expectation of the first error term can be easily estimated uniformly in $t \in[0, T]$ :

$$
\int_{\Omega^{n}}\left|\operatorname{Err}_{1}^{n}(t, \underline{\omega})\right| \mathrm{d} \mu_{t}^{n}=\mathcal{O}\left(n^{-1+\beta}\right) .
$$

By Lemma 1 there exists a smooth function $U(u, v)$ such that

$$
\partial_{\theta} U(u(\theta, \tau), v(\theta, \tau))=\Phi(u(\theta, \tau), v(\theta, \tau)), \quad \partial_{\tau} U(u(\theta, \tau), v(\theta, \tau))=\Psi(u(\theta, \tau), v(\theta, \tau)) .
$$

Thus $\operatorname{Err}_{2}^{n}(t)$ takes the form:

$$
\operatorname{Err}_{2}^{n}(t)=n^{3 \beta-1} \sum_{j \in \mathbb{T}^{n}} \partial_{x} U\left(n^{-\beta} \tilde{u}^{(n)}\left(t, \frac{j}{n}\right), n^{-\beta} \tilde{v}^{(n)}\left(t, \frac{j}{n}\right)\right),
$$


from which

$$
\operatorname{Err}_{2}(t)=\mathcal{O}\left(n^{-1+2 \beta}\right),
$$

uniformly for $t \in[0, T]$. From the previous bounds we have

$$
\begin{aligned}
& \int_{0}^{t} \int_{\Omega^{n}} n^{3 \beta} L^{n} \log \tilde{f}_{s}^{n}(\underline{\omega}) \mathrm{d} \mu_{s}^{n} \mathrm{~d} s \\
& =n^{-1+2 \beta} \int_{0}^{t} \int_{\Omega^{n}} \sum_{j \in \mathbb{T}^{n}}\left(\phi_{j}-\Phi\left(n^{-\beta} \tilde{u}_{j}^{(n)}, n^{-\beta} \tilde{v}_{j}^{(n)}\right)\right) \partial_{x} \tilde{\theta}^{(n)}\left(s, \frac{j}{n}\right) \\
& \quad+\left(\psi_{j}-\Psi\left(n^{-\beta} \tilde{u}_{j}^{(n)}, n^{-\beta} \tilde{v}_{j}^{(n)}\right)\right) \partial_{x} \tilde{\tau}^{(n)}\left(s, \frac{j}{n}\right) \mathrm{d} \mu_{s}^{n} \mathrm{~d} s+\mathcal{O}\left(n^{-1+2 \beta}\right) .
\end{aligned}
$$

In the next step we introduce the block averages. We will denote the block size with $l=l(n)$, it will be large microscopically, but small on the macroscopic scale. In the first computations we only assume $l \gg n^{2 \beta}$, the exact order of $l$ will only be determined at the end of the proof, after collecting all the error terms. For a local function $\kappa_{j}\left(j \in \mathbb{T}^{n}\right)$ we define its block average with

$$
\kappa_{j}^{l}:=\frac{1}{l} \sum_{i=0}^{l-1} \kappa_{j+i}
$$

By partial summation for a smooth function $\rho(x): \mathbb{T} \mapsto \mathbb{R}$ we have

$$
\left|\sum_{j \in \mathbb{T}^{n}} \kappa_{j} \rho\left(\frac{j}{n}\right)-\sum_{j \in \mathbb{T}^{n}} \kappa_{j}^{l} \rho\left(\frac{j}{n}\right)\right| \leqslant\left\|\partial_{x} \rho\right\|_{\infty}\left|\sum_{j \in \mathbb{T}^{n}} \kappa_{j}\right| \frac{l}{n},
$$

Using this with Lemma 3 we can replace $\phi_{j}, \psi_{j}$ in (44) with the respective block averages:

$$
\begin{aligned}
& \int_{0}^{t} \int_{\Omega^{n}} n^{3 \beta} L^{n} \log \tilde{f}_{s}^{n}(\underline{\omega}) \mathrm{d} \mu_{s}^{n} \mathrm{~d} s \\
& =n^{-1+2 \beta} \int_{0}^{t} \int_{\Omega^{n}} \sum_{j \in \mathbb{T}^{n}}\left\{\left(\phi_{j}^{l}-\Phi\left(n^{-\beta} \tilde{u}_{j}^{(n)}, n^{-\beta} \tilde{v}_{j}^{(n)}\right)\right) \partial_{x} \tilde{\theta}^{(n)}\left(s, \frac{j}{n}\right)\right. \\
& \left.\quad+\left(\psi_{j}^{l}-\Psi\left(n^{-\beta} \tilde{u}_{j}^{(n)}, n^{-\beta} \tilde{v}_{j}^{(n)}\right)\right) \partial_{x} \tilde{\tau}^{(n)}\left(s, \frac{j}{n}\right)\right\} \mathrm{d} \mu_{s}^{n} \mathrm{~d} s+\mathcal{O}\left(n^{\beta-1} l\right) .
\end{aligned}
$$

Finally, using Lemma 4 below (the one-block estimate), we replace the block averages $\phi_{j}^{l}, \psi_{j}^{l}$ by their 'local equilibrium value': $\Phi\left(\zeta_{j}^{l}, \eta_{j}^{l}\right)$ and $\Psi\left(\zeta_{j}^{l}, \eta_{j}^{l}\right)$, respectively:

$$
\begin{aligned}
& \int_{0}^{t} \int_{\Omega^{n}} n^{3 \beta} L^{n} \log \tilde{f}_{s}^{n}(\underline{\omega}) \mathrm{d} \mu_{s}^{n} \mathrm{~d} s \\
& =n^{-1+2 \beta} \int_{0}^{t} \int_{\Omega^{n}} \sum_{j \in \mathbb{T}^{n}}\left(\Phi\left(\zeta_{j}^{l}, \eta_{j}^{l}\right)-\Phi\left(n^{-\beta} \tilde{u}_{j}^{(n)}, n^{-\beta} \tilde{v}_{j}^{(n)}\right)\right) \partial_{x} \tilde{\theta}^{(n)}\left(s, \frac{j}{n}\right) \\
& \quad+\left(\Psi\left(\zeta_{j}^{l}, \eta_{j}^{l}\right)-\Psi\left(n^{-\beta} \tilde{u}_{j}^{(n)}, n^{-\beta} \tilde{v}_{j}^{(n)}\right)\right) \partial_{x} \tilde{\tau}^{(n)}\left(s, \frac{j}{n}\right) \mathrm{d} \mu_{s}^{n} \mathrm{~d} s+\mathcal{O}\left(n^{\beta-1} l \vee n^{-1-\beta} l^{3} \vee l^{-1}\right) .
\end{aligned}
$$


Lemma 4 (One block estimate).

$$
\begin{aligned}
& \frac{1}{n} \int_{0}^{t} \int_{\Omega^{n}} \sum_{j \in \mathbb{T}^{n}}\left|\phi_{j}^{l}-\Phi\left(\zeta_{j}^{l}, \eta_{j}^{l}\right)\right| \mathrm{d} \mu_{s}^{n} \mathrm{~d} t \leqslant C\left(n^{-1-3 \beta} l^{3}+l^{-1}\right), \\
& \frac{1}{n} \int_{0}^{t} \int_{\Omega^{n}} \sum_{j \in \mathbb{T}^{n}}\left|\psi_{j}^{l}-\Psi\left(\zeta_{j}^{l}, \eta_{j}^{l}\right)\right| \mathrm{d} \mu_{s}^{n} \mathrm{~d} t \leqslant C\left(n^{-1-3 \beta} l^{3}+l^{-1}\right) .
\end{aligned}
$$

The proof relies on the spectral gap condition (D). It uses the Feynman-Kac formula, the Raleigh-Schrödinger perturbation technique and the 'equivalence of ensembles' (see the Appendix of [8] for all three). A detailed proof can be found in [14] for the one component case which can be easily adapted for our purposes.

\section{Remarks.}

1. If instead of the condition (D) we have the stronger log-Sobolev inequality $\left(\mathrm{D}^{\prime}\right)$, then the previous lemma may be strengthened: it holds with the bound $C\left(n^{-1-3 \beta} l^{2}+l^{-1}\right)$, see [16].

2. We need the following form of the 'equivalence of ensembles'. Let $l$ be a fixed positive integer. Then the distribution of the first $l$ marginals of the microcanonical measure $\pi_{Z, N}^{n}$ is asymptotically the same as the canonical distribution $\pi_{Z / n, N / n}^{l}$ and their total variation distance is $\mathcal{O}(1 / n)$ uniformly in $Z, N$. In the case of one conserved quantity this is proved in the Appendix of [8], the proof relies on the local central limit theorem and the asymptotic expansion for i.i.d. lattice random variables. Since similar strong results are available for i.i.d. lattice random vectors (see [2], Chapter 5), one can transform the proof of [8] for our case. It is worth noting however, that for the specific models considered in Subsection 2.5 we do not need this general theory. For the two-lane models the two conserved quantities are independent with respect to the canonical measures which means that the microcanonical measures also factorize. Thus the equivalence of ensembles follows from the one conserved quantity case.

For the $\{-1,0,1\}$-model the microcanonical measure $\pi_{Z, N}^{n}$ is the uniform measure on all configurations where the number of -1 's is $\frac{1}{2}(n-Z-N)$, the number 0's is $N$ and the number of 1 's is $\frac{1}{2}(Z+n-N)$. For the equivalence of ensembles one essentially needs to prove that sampling without replacement $l$ times and sampling with replacement $l$ times from these $n$ symbols will result in two distributions which are sufficiently close in total variation (if $n$ is large and $l$ is kept fixed). This can be readily shown by a straightforward (although a bit lengthy) computation.

\subsection{Estimating the second term of (38)}

Performing the time-derivation we obtain:

$$
n^{-1+2 \beta} \partial_{t} \log \tilde{f}_{t}^{n}=\frac{1}{n} \sum_{j \in \mathbb{T}^{n}}\left\{\left(n^{\beta} \zeta_{j}-\tilde{u}_{j}^{(n)}\right) \partial_{t} \tilde{\theta}^{(n)}\left(t, \frac{j}{n}\right)+\left(n^{\beta} \eta_{j}-\tilde{v}_{j}^{(n)}\right) \partial_{t} \tilde{\tau}^{(n)}\left(t, \frac{j}{n}\right)\right\} .
$$

By the definitions of $\tilde{u}^{(n)}, \tilde{v}^{(n)}$, and taking the Taylor-expansion of $\Phi(u, v)$ around $(0,0)$ we readily get that

$$
\begin{aligned}
\partial_{t} \tilde{u}^{(n)}(t, x)= & \sigma_{t}\left(t, x-\lambda n^{\beta} t\right)-\lambda n^{\beta} \sigma_{x}\left(t, x-\lambda n^{\beta} t\right)-\lambda \bar{\sigma}_{x_{1}}\left(t, x-\lambda n^{\beta} t, x-\mu n^{\beta} t\right) \\
& -\mu \bar{\sigma}_{x_{2}}\left(t, x-\lambda n^{\beta} t, x-\mu n^{\beta} t\right)+\mathcal{O}\left(n^{-\beta}\right),
\end{aligned}
$$

and 


$$
\begin{aligned}
n^{2 \beta} \partial_{x} & \Phi\left(n^{-\beta} \tilde{u}^{(n)}(x, t), n^{-\beta} \tilde{v}^{(n)}(x, t)\right) \\
= & \lambda n^{\beta} \sigma_{x}\left(t, x-\lambda n^{\beta} t\right)+\lambda \bar{\sigma}_{x_{1}}\left(t, x-\lambda n^{\beta} t, x-\mu n^{\beta} t\right)+\lambda \bar{\sigma}_{x_{2}}\left(t, x-\lambda n^{\beta} t, x-\mu n^{\beta} t\right) \\
& +\partial_{x}\left(\frac{1}{2} a_{1} \sigma\left(t, x-\lambda n^{\beta} t\right)^{2}+c_{\delta} a_{2} \sigma\left(t, x-\lambda n^{\beta} t\right)\right) \\
& +\partial_{x}\left(a_{2} \sigma\left(t, x-\lambda n^{\beta} t\right)\left(\delta\left(t, x-\mu n^{\beta} t\right)-c_{\delta}\right)+\frac{1}{2} a_{3} \delta\left(t, x-\mu n^{\beta} t\right)^{2}\right)+\mathcal{O}\left(n^{-\beta}\right),
\end{aligned}
$$

with uniform error terms. ( $\bar{\sigma}_{x_{1}}$ and $\bar{\sigma}_{x_{2}}$ are the partial derivatives of $\bar{\sigma}\left(t, x_{1}, x_{2}\right)$ with respect to the second and third variable.) Adding up these equations and checking the definitions for $\sigma, \delta, \bar{\sigma}$ we see that all the significant terms on the right-hand side cancel to give:

$$
\partial_{t} \tilde{u}^{(n)}+\partial_{x}\left(n^{2 \beta} \Phi\left(n^{-\beta} \tilde{u}^{(n)}, n^{-\beta} \tilde{v}^{(n)}\right)\right)=\mathcal{O}\left(n^{-\beta}\right) .
$$

Similarly,

$$
\partial_{t} \tilde{v}^{(n)}+\partial_{x}\left(n^{2 \beta} \Psi\left(n^{-\beta} \tilde{u}^{(n)}, n^{-\beta} \tilde{v}^{(n)}\right)\right)=\mathcal{O}\left(n^{-\beta}\right) .
$$

From (48) and (49):

$$
\begin{aligned}
\partial_{t} \tilde{\theta}^{(n)} & =\theta_{u} \partial_{t} \tilde{u}^{(n)}+\theta_{v} \partial_{t} \tilde{v}^{(n)} \\
& =-n^{2 \beta} \theta_{u} \partial_{x}\left(\Phi\left(n^{-\beta} \tilde{u}^{(n)}, n^{-\beta} \tilde{v}^{(n)}\right)\right)-n^{2 \beta} \theta_{v} \partial_{x}\left(\Psi\left(n^{-\beta} \tilde{u}^{(n)}, n^{-\beta} \tilde{v}^{(n)}\right)\right)+\mathcal{O}\left(n^{-\beta}\right) \\
& =-n^{\beta}\left(\theta_{u} \Phi_{u}+\theta_{v} \Psi_{u}\right) \partial_{x} \tilde{u}^{(n)}-n^{\beta}\left(\theta_{u} \Phi_{v}+\theta_{v} \Psi_{v}\right) \partial_{x} \tilde{v}^{(n)}+\mathcal{O}\left(n^{-\beta}\right) \\
& =-n^{\beta}\left(\theta_{u} \Phi_{u}+\tau_{u} \Psi_{u}\right) \partial_{x} \tilde{u}^{(n)}-n^{\beta}\left(\theta_{v} \Phi_{u}+\tau_{v} \Psi_{u}\right) \partial_{x} \tilde{v}^{(n)}+\mathcal{O}\left(n^{-\beta}\right) \\
& =-n^{\beta} \Phi_{u} \partial_{x} \tilde{\theta}^{(n)}-n^{\beta} \Psi_{u} \partial_{x} \tilde{\tau}^{(n)}+\mathcal{O}\left(n^{-\beta}\right) .
\end{aligned}
$$

In the fourth line we used $\tau_{u}=\theta_{v}$ and also Lemma 1. To simplify notations, we omitted the arguments $(t, x)$ from the functions $\tilde{\theta}^{(n)}, \tilde{\tau}^{(n)}, \tilde{u}^{(n)}, \tilde{v}^{(n)}$, and the arguments $\left(n^{-\beta} \tilde{u}^{(n)}, n^{-\beta} \tilde{v}^{(n)}\right)$ from all the partial derivatives of $\theta, \tau, \Phi, \Psi$ with respect to $u, v$. Similarly,

$$
\partial_{t} \tilde{\tau}^{(n)}=-n^{\beta} \Phi_{v} \partial_{x} \tilde{\theta}^{(n)}-n^{\beta} \Psi_{v} \partial_{x} \tilde{\tau}^{(n)}+\mathcal{O}\left(n^{-\beta}\right) .
$$

Hence from (47):

$$
\begin{aligned}
n^{-1+2 \beta} \partial_{t} \log \tilde{f}_{t}^{n}= & -n^{-1+2 \beta} \sum_{j \in \mathbb{T}^{n}}\left\{\left(\zeta_{j}-n^{-\beta} \tilde{u}_{j}^{(n)}\right) \Phi_{u}\left(n^{-\beta} \tilde{u}_{j}^{(n)}, n^{-\beta} \tilde{v}_{j}^{(n)}\right) \partial_{x} \tilde{\theta}^{(n)}\left(t, \frac{j}{n}\right)\right. \\
& +\left(\zeta_{j}-n^{-\beta} \tilde{u}_{j}^{(n)}\right) \Psi_{u}\left(n^{-\beta} \tilde{u}_{j}^{(n)}, n^{-\beta} \tilde{v}_{j}^{(n)}\right) \partial_{x} \tilde{\tau}^{(n)}\left(t, \frac{j}{n}\right) \\
& +\left(\eta_{j}-n^{-\beta} \tilde{v}_{j}^{(n)}\right) \Phi_{v}\left(n^{-\beta} \tilde{u}_{j}^{(n)}, n^{-\beta} \tilde{v}_{j}^{(n)}\right) \partial_{x} \tilde{\theta}^{(n)}\left(t, \frac{j}{n}\right) \\
& \left.+\left(\eta_{j}-n^{-\beta} \tilde{v}_{j}^{(n)}\right) \Psi_{v}\left(n^{-\beta} \tilde{u}_{j}^{(n)}, n^{-\beta} \tilde{v}_{j}^{(n)}\right) \partial_{x} \tilde{\tau}^{(n)}\left(t, \frac{j}{n}\right)\right\}+\operatorname{Err}_{3}(t, \underline{\omega})
\end{aligned}
$$

where

$$
\operatorname{Err}_{3}(t, \underline{\omega})=\frac{1}{n} \sum_{j \in \mathbb{T}^{n}}\left(\zeta_{j}-n^{-\beta} \tilde{u}_{j}^{(n)}\right) b_{j}(t)+\left(\eta_{j}-n^{-\beta} \tilde{v}_{j}^{(n)}\right) c_{j}(t)
$$

and $b_{j}(t)$ and $c_{j}(t)$ are uniformly bounded constants. Using Lemma 3 we get that

$$
\int_{\Omega^{n}}\left|\operatorname{Err}_{3}(t, \underline{\omega})\right| \mathrm{d} \mu_{t}^{n}=\mathcal{O}\left(n^{-\beta}\right) .
$$


We can exchange $\zeta_{j}, \eta_{j}$ with their block-averages $\zeta_{j}^{l}, \eta_{j}^{l}$ (as in the previous subsection) which (after the timeintegration) gives the following estimate:

$$
\begin{aligned}
\int_{0}^{t} \int_{\Omega^{n}} n^{-1+2 \beta} \partial_{t} \log \tilde{f}_{s}^{n} \mathrm{~d} \mu_{s}^{n} \mathrm{~d} s= & -\int_{0}^{t} \int_{\Omega^{n}} n^{-1+2 \beta} \sum_{j \in \mathbb{T}^{n}}\left\{\left(\zeta_{j}^{l}-n^{-\beta} \tilde{u}_{j}^{(n)}\right) \Phi_{u}\left(n^{-\beta} \tilde{u}_{j}^{(n)}, n^{-\beta} \tilde{v}_{j}^{(n)}\right) \partial_{x} \tilde{\theta}^{(n)}\left(s, \frac{j}{n}\right)\right. \\
& +\left(\zeta_{j}^{l}-n^{-\beta} \tilde{u}_{j}^{(n)}\right) \Psi_{u}\left(n^{-\beta} \tilde{u}_{j}^{(n)}, n^{-\beta} \tilde{v}_{j}^{(n)}\right) \partial_{x} \tilde{\tau}^{(n)}\left(s, \frac{j}{n}\right) \\
& +\left(\eta_{j}^{l}-n^{-\beta} \tilde{v}_{j}^{(n)}\right) \Phi_{v}\left(n^{-\beta} \tilde{u}_{j}^{(n)}, n^{-\beta} \tilde{v}_{j}^{(n)}\right) \partial_{x} \tilde{\theta}^{(n)}\left(s, \frac{j}{n}\right) \\
& \left.+\left(\eta_{j}^{l}-n^{-\beta} \tilde{v}_{j}^{(n)}\right) \Psi_{v}\left(n^{-\beta} \tilde{u}_{j}^{(n)}, n^{-\beta} \tilde{v}_{j}^{(n)}\right) \partial_{x} \tilde{\tau}^{(n)}\left(s, \frac{j}{n}\right)\right\} \mathrm{d} \mu_{s}^{n} \mathrm{~d} s \\
& +\mathcal{O}\left(n^{-\beta} \vee n^{\beta-1} l\right) .
\end{aligned}
$$

\subsection{Block replacement}

For a function $\Upsilon(u, v)$ we denote

$$
\mathrm{R}_{\Upsilon}\left(u_{1}, v_{1} ; u_{2}, v_{2}\right):=\Upsilon\left(u_{1}, v_{1}\right)-\Upsilon\left(u_{2}, v_{2}\right)-\Upsilon_{u}\left(u_{2}, v_{2}\right)\left(u_{1}-u_{2}\right)-\Upsilon_{v}\left(u_{2}, v_{2}\right)\left(v_{1}-v_{2}\right) .
$$

Collecting the estimates of the previous subsections and noting that the functions $\left|\partial_{x} \tilde{\theta}^{(n)}\right|,\left|\partial_{x} \tilde{\tau}^{(n)}\right|$ are uniformly bounded in $[0, T] \times \mathbb{T}$ we have

$$
\begin{aligned}
H\left(\mu_{t}^{n} \mid \tilde{v}_{t}^{n}\right)-H\left(\mu_{t}^{n} \mid \tilde{v}_{0}^{n}\right) \leqslant & C n^{2 \beta-1} \int_{0}^{t} \int_{\Omega^{n}} \sum_{j \in \mathbb{T}^{n}}\left(\left|\mathrm{R}_{\Phi}\left(\zeta_{j}^{l}, \eta_{j}^{l} ; n^{-\beta} \tilde{u}^{(n)}\left(s, \frac{j}{n}\right), n^{-\beta} \tilde{v}^{(n)}\left(s, \frac{j}{n}\right)\right)\right|\right. \\
& \left.+\left|\mathrm{R}_{\Psi}\left(\zeta_{j}^{l}, \eta_{j}^{l} ; n^{-\beta} \tilde{u}^{(n)}\left(s, \frac{j}{n}\right), n^{-\beta} \tilde{v}^{(n)}\left(s, \frac{j}{n}\right)\right)\right|\right) \mathrm{d} \mu_{s}^{n} \mathrm{~d} s \\
& +\mathcal{O}\left(n^{-\beta} \vee n^{\beta-1} l \vee n^{-1-\beta} l^{3}\right) .
\end{aligned}
$$

The second derivatives of $\Phi$ and $\Psi$ are bounded in $\mathcal{D}$ thus

$$
\begin{aligned}
& \left|\mathrm{R}_{\Phi}\left(\zeta_{j}^{l}, \eta_{j}^{l} ; n^{-\beta} \tilde{v}^{(n)}\left(s, \frac{j}{n}\right), n^{-\beta} \tilde{u}^{(n)}\left(s, \frac{j}{n}\right)\right)\right|+\left|\mathrm{R}_{\Psi}\left(\zeta_{j}^{l}, \eta_{j}^{l} ; n^{-\beta} \tilde{v}^{(n)}\left(s, \frac{j}{n}\right), n^{-\beta} \tilde{u}^{(n)}\left(s, \frac{j}{n}\right)\right)\right| \\
& \leqslant C\left(\left(\zeta_{j}^{l}-n^{-\beta} \tilde{u}^{(n)}\left(s, \frac{j}{n}\right)\right)^{2}+\left(\eta_{j}^{l}-n^{-\beta} \tilde{v}^{(n)}\left(s, \frac{j}{n}\right)\right)^{2}\right),
\end{aligned}
$$

which means that it is sufficient to estimate

$$
n^{2 \beta-1} \int_{\Omega^{n}} \sum_{j \in \mathbb{T}^{n}}\left(\zeta_{j}^{l}-n^{-\beta} \tilde{u}^{(n)}\left(t, \frac{j}{n}\right)\right)^{2} \mathrm{~d} \mu_{t}^{n}+n^{2 \beta-1} \int_{\Omega^{n}} \sum_{j \in \mathbb{T}^{n}}\left(\eta_{j}^{l}-n^{-\beta} \tilde{v}^{(n)}\left(t, \frac{j}{n}\right)\right)^{2} \mathrm{~d} \mu_{t}^{n}
$$

uniformly in $t$. We estimate the first expression, the other will follow the same way. We denote

$$
\hat{\zeta}_{j}^{l}:=\hat{\zeta}_{j}^{l}(t, \underline{\omega})=\frac{1}{l} \sum_{i=0}^{l-1}\left(\zeta_{j+i}-n^{-\beta} \tilde{u}^{(n)}\left(t, \frac{j+i}{n}\right)\right) .
$$

Since $\partial_{x} \tilde{u}^{(n)}(t, x)$ is uniformly bounded for $(t, x) \in[0, T] \times \mathbb{T}$, we have

$$
\left(\hat{\zeta}_{j}^{l}\right)^{2}-\left(\zeta_{j}^{l}-n^{-\beta} \tilde{u}_{j}^{(n)}\right)^{2}=\mathcal{O}\left(n^{-\beta-1} l\right)
$$


uniformly in $j \in \mathbb{T}^{n}, t \in[0, T]$ and it is enough to estimate

$$
n^{2 \beta-1} \int_{\Omega^{n}} \sum_{j \in \mathbb{T}^{n}}\left(\hat{\zeta}_{j}^{l}\right)^{2} \mathrm{~d} \mu_{t}^{n}
$$

Applying the entropy inequality with respect to the time-dependent reference measure $\tilde{v}_{t}^{n}$ and using Hölder's inequality:

$$
n^{2 \beta-1} \int_{\Omega^{n}} \sum_{j \in \mathbb{T}^{n}}\left(\hat{\zeta}_{j}^{l}\right)^{2} \mathrm{~d} \mu_{t}^{n} \leqslant \frac{1}{\gamma} n^{2 \beta-1} H\left(\mu_{t}^{n} \mid \tilde{v}_{t}^{n}\right)+\frac{1}{\gamma} l^{-1} n^{2 \beta-1} \sum_{j \in \mathbb{T}^{n}} \log \mathbf{E}_{\tilde{v}_{t}^{n}} \exp \left(\gamma l\left(\hat{\zeta}_{j}^{l}\right)^{2}\right),
$$

for any $\gamma>0$. D is compact, $\zeta$ is bounded thus there exists a positive constant $C$ such that

$$
\log \mathbf{E}_{u, v} \exp ((\zeta-u) y) \leqslant C y^{2}
$$

for all $(u, v) \in \mathcal{D}$ and $y \in \mathbb{R}$. Thus as a consequence of Lemma 5 below, there exists a small, but fixed $\gamma>0$ independent of $n$ and $l$ for which

$$
\frac{1}{n} \sum_{j \in \mathbb{T}^{n}} \log \mathbf{E}_{\tilde{v}_{t}^{n}} \exp \left(\gamma l\left(\hat{\zeta}_{j}^{l}\right)^{2}\right)<1
$$

Substituting this into (57):

$$
n^{2 \beta-1} \int_{\Omega^{n}} \sum_{j \in \mathbb{T}^{n}}\left(\zeta_{j}^{l}-n^{-\beta} \tilde{u}^{(n)}\left(t, \frac{j}{n}\right)\right)^{2} \mathrm{~d} \mu_{t}^{n}<C n^{2 \beta-1} H\left(\mu_{t}^{n} \mid \tilde{v}_{t}^{n}\right)+\mathcal{O}\left(n^{2 \beta} l^{-1}\right) .
$$

Collecting all the estimates, from (56) we get

$$
n^{2 \beta-1}\left(H\left(\mu_{t}^{n} \mid \tilde{v}_{t}^{n}\right)-H\left(\mu_{0}^{n} \mid \tilde{v}_{0}^{n}\right)\right) \leqslant C n^{2 \beta-1} \int_{0}^{t} H\left(\mu_{s}^{n} \mid \tilde{\nu}_{s}^{n}\right) \mathrm{d} s+\mathcal{O}\left(n^{-\beta} \vee n^{\beta-1} l \vee n^{-1-\beta} l^{3} \vee n^{2 \beta} l^{-1}\right)
$$

Since $\beta \in\left(0, \frac{1}{5}\right)$, we can choose $l$ to satisfy

$$
n^{2 \beta} \ll l \ll n^{(1+\beta) / 3}
$$

which makes the error term o(1). The theorem now follows via Lemma 2 and the Grönwall inequality as discussed before.

If we have the logarithmic-Sobolev condition, and thus a stronger version of Lemma 4, then $l$ can be chosen with $n^{2 \beta} \ll l \ll n^{(1+\beta) / 2}$ to make all the error terms o(1). Thus, in that case the theorem holds with $\beta \in\left(0, \frac{1}{3}\right)$.

The proof of Lemma 5 below can be found in [14] or [16].

Lemma 5. Suppose $\xi_{1}, \xi_{2}, \ldots$ are independent random variables with $\mathbf{E} \xi_{i}=0$ for which

$$
\log \mathbf{E} \exp \left(y \xi_{i}\right) \leqslant C y^{2}
$$

with a positive constant $C$ independent of $i$ and $y$. Then there exists a small positive constants $\gamma$ depending only on $C$ such that

$$
\log \mathbf{E} \exp \left(\gamma l\left(\xi_{i}^{l}\right)^{2}\right)<1 .
$$




\section{Acknowledgements}

The author thanks Bálint Tóth for introducing him into the theory of hydrodynamic limits, and particularly to the problem of the present paper. He also thanks Fraydoun Rezakhanlou for drawing his attention to the paper [4] and the method of geometric optics.

This work was partially supported by the Hungarian Scientific Research Fund (OTKA) grants no. T037685 and TS40719.

\section{References}

[1] M. Balázs, Growth fluctuations in interface models, Ann. Inst. H. Poincaré Probab. Statist. 39 (2003) 639-685.

[2] R.N. Bhattacharya, R. Ranga Rao, Normal Approximation and Asymptotic Expansions, Wiley, 1976.

[3] C. Cocozza, Processus des misanthropes, Z. Wahrscheinlichkeitstheorie Verw. Gebiete 70 (1985) 509-523.

[4] R.J. DiPerna, A. Majda, The validity of nonlinear geometric optics for weak solutions of conservation laws, Commun. Math. Phys. 98 (1985) 313-347.

[5] R. Esposito, R. Marra, H.T. Yau, Diffusive limit of asymmetric simple exclusion, Rev. Math. Phys. 6 (1994) $1233-1267$.

[6] J. Fritz, B. Tóth, Derivation of the Leroux system as the hydrodynamic limit of a two-component lattice gas, Commun. Math. Phys. 249 (2004) 1-27.

[7] J.K. Hunter, J.B. Keller, Weakly nonlinear high frequency waves, Commun. Pure Appl. Math. 36 (1983) 547-569.

[8] C. Kipnis, C. Landim, Scaling Limits of Interacting Particle Systems, Springer, 1999.

[9] C. Landim, S. Sethuraman, S.R.S. Varadhan, Spectral gap for zero range dynamics, Ann. Probab. 24 (1986) 1871-1902.

[10] S. Olla, S.R.S. Varadhan, H.T. Yau, Hydrodynamical limit for Hamiltonian system with weak noise, Commun. Math. Phys. 155 (1993) 523-560.

[11] V. Popkov, G.M. Schütz, Shocks and excitation dynamics in driven diffusive two channel systems, J. Statist. Phys. 112 (2003) 523-540.

[12] F. Rezakhanlou, Microscopic structure of shocks in one conservation laws, Ann. Inst. H. Poincaré Anal. Non Lineaire 12 (1995) 119-153.

[13] T. Seppäläinen, Perturbation of the equilibrium for a totally asymmetric stick process in one dimension, Ann. Probab. 29 (2001) 176-204.

[14] B. Tóth, B. Valkó, Between equilibrium fluctuations and Eulerian scaling. Perturbation of equilibrium for a class of deposition models, J. Statist. Phys. 109 (2002) 177-205.

[15] B. Tóth, B. Valkó, Onsager relations and Eulerian hydrodynamic limit for systems with several conservation laws, J. Statist. Phys. 112 (2003) 497-521.

[16] B. Tóth, B. Valkó, Perturbation of singular equilibria of hyperbolic two-component systems: a universal hydrodynamic limit, Commun. Math. Phys. 256 (2005) 111-157.

[17] H.T. Yau, Logarithmic Sobolev inequality for generalized simple exclusion processes, Probability Theory Related Fields 109 (1997) 507538. 